\title{
JomAR Purchasing Furniture in Augmented Reality Experiences
}

\author{
J.R. Prasojo, UCSI University, Malaysia \\ P.S. JosephNg, UCSI University, Malaysia
}

\begin{abstract}
In the modern world, online shopping is a common activity that people do. They buy all things, even large-size products such as furniture. People used to measure the product traditionally using a tape measure. However, people can take advantages of the virtual reality products such as augmented reality. This study used mixed methodology involving 105 respondents. The augmented reality will reduce the cost since the customer has no need to measure traditionally and increase efficiency to buy the product. Additionally, the customer will have more trust towards the seller. Augmented reality can help the user to understand the product and match the product with the user's surroundings. This augmented reality will potentially grow and adapt to the user needs as the user might explore more with the image projection.
\end{abstract}

\section{KEYWORDS}

Augmented Reality, Furniture, Mobile Shopping, Online Shopping, Virtual

\section{INTRODUCTION}

Modern people now able to purchase a thing from their phone. The concept of buying product from the phone only can happen because of the invention of the internet. Earlier internet only can be used to access data and information such as an encyclopedia or other useful source. As time goes, some people have the idea to help people who want to buy the product but limited to the condition of the surroundings. This idea now called online shopping and commonly used nowadays. Modern online shopping now called e-commerce which meaning is electronic commerce. The earlier invention of electronic commerce was the website based online shop. This web-based online shop becomes popular and can fulfil a lot of people need. The main reason for this development is to create an opportunity so that people do not need to travel only to buy something.

Electronic commerce becomes popular as people can purchase the product without having to travel (Chiu, W. and Cho, H. 2019). This online shopping experience gives numerous advantages to people. The foremost seen advantage is that people do not need to go outside or even travel to the place which provides the product that people want to buy (Gupta, P. and Dubey, A. 2017). Another advantage of this electronic commerce is that people do not need to queue while waiting for the checkout process after choosing the product. Through electronic commerce, people can just choose their product and checkout as fast as possible. The checkout process also relatively fast since electronic commerce is built-in with online banking. The carrying process of the product also becomes easier. The person does not need to carry the product while the product already handled by the courier from the electronic commerce provider. The user may have a delay in receiving the product, but it is the price to pay for convenience.

\section{DOI: 10.4018 /IJBSA.287110}

This article published as an Open Access article distributed under the terms of the Creative Commons Attribution License (http://creativecommons.org/licenses/by/4.0/) which permits unrestricted use, distribution, and production in any medium, provided the author of the original work and original publication source are properly credited. 
RQ1: What effect could when changing from conventional e-commerce to augmented-reality e-commerce? RQ2: How augmented reality can be implemented in mobile commerce?

RQ3: How augmented reality can be impactful to the customer?

The development of electronic commerce continues as people now travels through space frequently. Modern people become busier and have a lot of complexity. One people can have more than 2 jobs, this will force them to travel a lot in one day. Due to this change of habit, electronic commerce becomes accessible on mobile devices (Engelmann, T., Wallstein, S., \& Hitzler, D. 2019). This now called mobile commerce. People not only can access the store from the computer but also, they can access the online shop from their mobile devices. This becomes a solution for the people who do not have time to stay and purchase daily groceries from their computer or laptop. This purchase action can be done through their phone and this mobile commerce will send what the person wants directly. The process now becomes easier and simpler since the person only need to access their phone (Kang, C.M., JosephNg, P.S. \& Issa, K. 2015),

The product purchased from one individual can have a wide of variety (Wakil, K., Alyari, F., Ghasvari, M., Lesani, Z. and Rajabion, L. 2019). Ecommerce also provides not only daily groceries but also other product such as car equipment, electronics, clothing, and even furniture (Trabelsi-Zoghlami, A. and Touzani, M. 2019). People sometimes facing difficulties in buying the product. They will sometimes receive a product that is not correct and not fulfilling their needs. Some people can receive great dissatisfaction because the product received is not the same as stated on mobile commerce.

In the current conventional e-commerce market, the application only shows the picture of the product rather than the real projection of the object. This will lead to some difference in the size of the product. In this application, the concern mainly on the large size product which are furniture and home electronic devices. In this category, people will hardly find to check the size compare to the real-life environment and will lead to less satisfaction with the product and services.

This application will have a function that utilizes the camera of the smartphone to extend the picture of the item inside the applications. This picture comes from the 3D model of the genuine item to contrast it and the current condition in the genuine area (Beck, J., Rainoldi, M. and Egger, R. (2019). The applications will decide the state of the real environmental factors to ensure that the extended picture is clear and have a comparative measurement. It will raise the size of the environmental factors region and will make a virtual picture of the item on the smartphone.

The simple user interface will create a better understanding for the user as they can easily use the application as a common electronic commerce application (Reynolds, J. 2015). The main menu of the application will provide all the product sold by the seller and the details page also explain every detail of the product. Moreover, the application also provides a login and register feature to give the user a better system inside the application. This apps aims to answer this research question provided in Table 1.

There are three research questions and to reduce the scope, the research will be conducted among university students. The research objective will answer each research question in Table 2.

Based on the research objective (Table 2), this mobile app aims to increase people's satisfaction in buying products online while the hypothesis is described in Table 3

H1: The company branding will increase due to the customer interest in augmented reality.

As the augmented reality is currently a new feature that implements in e-commerce, the customer will be interested to try the augmented reality feature (Haenlein, M. and Kaplan, A.M. 2009). This new feature will attract more people to not even try the feature but also buy the product from the company. 
RO1: To improve the branding of the company using the augmented reality feature

RO2: To implement augmented reality system on mobile commerce application.

RO3: To create a better user perspective about the product through augmented reality

Table 3. Hypothesis

H1: The company branding will increase due to the customer interest in augmented reality.

H2: The Augmented Reality feature will be implemented in mobile commerce application.

H3: The customer will gain a better understanding of the product through augmented reality

H2: The Augmented Reality feature will be implemented in mobile commerce application.

The presence of AR in an app leads to stronger positive affective responses compared with nonaugmented apps (Yaoyuneyong, G., Foster, J., Johnson, E. and Johnson, D. 2016, Javornik, A. 2016b). AR show how the presentation of the product before the customer receives the product. This will help to reduce the risk of different products from the real condition product compare to the picture of the product from online shopping.

H3: The customer will gain a better understanding of the product through augmented reality

The customer purchase decision is influenced visually (AdrianChin, Y.K., JosephNg, P. S., Shibghatullah, A. S. and Loh, Y. F. 2020, AdrianChin, Y.K.; JosephNg, P.S.; Eaw, H.C.; Loh, Y.F. \& Shibghatullah, A.S. 2020, Vivilyana, V. et al. 2020, Vica, V. et al. 2020) The augmented reality provides the feature for the customer to check the image of the product before they decide to buy the product. This feature will be useful as the customer can understand how the product matches the surroundings and their needs.

From the table 4 we can see that both the developed application, JomFurniReal, and other store has similarity in basic electronic commerce. However, not all store has the feature to augmented reality view which from here the customer can see the product beforehand. Even the store has augmented reality implementation the store may only have the feature to showcase the product and has no purchase feature in the application. This developed application will have benefits to prevent user dissatisfaction because of the product difference in dimension and specification.

Table 4. Feature comparison with other furniture stores

\begin{tabular}{|l|l|l|}
\hline Features & JomFurniReal & Other Store \\
\hline Login and Register & $/$ & $/$ \\
\hline Product Specification Info & $/$ & $/$ \\
\hline List of products & $/$ & $/$ \\
\hline AR view & $/$ & partially \\
\hline Purchase product & $/$ & partially \\
\hline
\end{tabular}




\section{LEGACY SHOPPING LITERATURE}

In the modern world, people may use augmented reality frequently as the technology keep moving and developing. The application of the augmented reality in the world could be seen in many industries such as recreational area which are 2D or 3D video games Watson, A., Alexander, B. and Salavati, L. (2018). The application of augmented reality in business is quite limited due to the importance of using it is not present. While the needs are not rising, the business area should consider augmented reality as an innovation to expand the business and give customer more option in purchasing product (Roxo, M. T., \& Brito, P. Q. 2018). The business area specifically the furniture industry would be able to utilize augmented reality as the technology will fulfil the customer expectation of the product. This expectation is important since some of the customers would have no understanding of the product unless they see the product in person. The augmented reality which has the function to project 3D image will come as a solution to create a better understanding for the customer of the product (Malaysian Communications and Multimedia Commission 2018).

The previous study limited the function of augmented reality as a platform to increase the user perspective in the virtual world (Seidl D., 2018). The study suggests that user willing to purchase the product when they see in the virtual world (Yoo, S.C. and Eastin, M.S. 2016). This study is a core of people perspective in the virtual world. Additionally, it provides insight into how people respond to the virtual world. However, the previous study would require certain hardware that advance enough for the normal user to afford and more complicated. The previous study also limits to cover only virtual reality which can be more advanced compare to the augmented reality (Chang, I.C., Liu, C.C. and Chen, K. 2014), Virtual reality requires VR hardware and a powerful computing unit to run. The complication of VR is not suitable to be implemented in the e-commerce area since some of the potential customers is not that aware of the technology and hardware. Meanwhile, augmented reality requires less hardware and less powerful computing unit. Thus, the augmented reality would be more useful to the user. The study will take advantage of the augmented reality function which is 3D image projection and apply it to common e-commerce (Vernette, E. 2018). The implementation of the augmented reality in eCommerce will increase the efficiency of the business life cycle and increase customer trust towards the business (Domina T., Lee S.-E., MacGillivray M., 2012, Hussy W., Schreier M., Echterhoff G., 2015, Cowan, K. and Ketron, S. 2019).

\section{METHODOLOGY}

The primary research method for this study is the mixed methodology which mixes the qualitative and quantitative data collection to answer the question from the target audience more effectively. The participant will consist of people who had experience in buying online especially those who buy furniture through the online store. There is two part for the data collection in the study, which are the online questionnaire and online interview are shown in Figure 1.

\section{RESULTS AND FINDINGS}

According to the data collected from the survey, most of the respondent said that they ever used the online store to purchase the product and only $1 \%$ of the respondent said they never use the online store. And the followed question asked about whether the person had purchase furniture or large-size product through the online store. The answer is more than $64 \%$ of the respondent said they never buy furniture product online. This could happen since the majority of people would not trust online store to buy furniture product.

The following question asking the respondent experience in buying a product whether they ever experienced the different appearance of the product before and after they purchase it online. The result was more than $50 \%$ of the respondent had experience product difference after the product arrived. The 
Figure 1. Mixed methodology diagram (P.S. JosephNg 2021-2011)

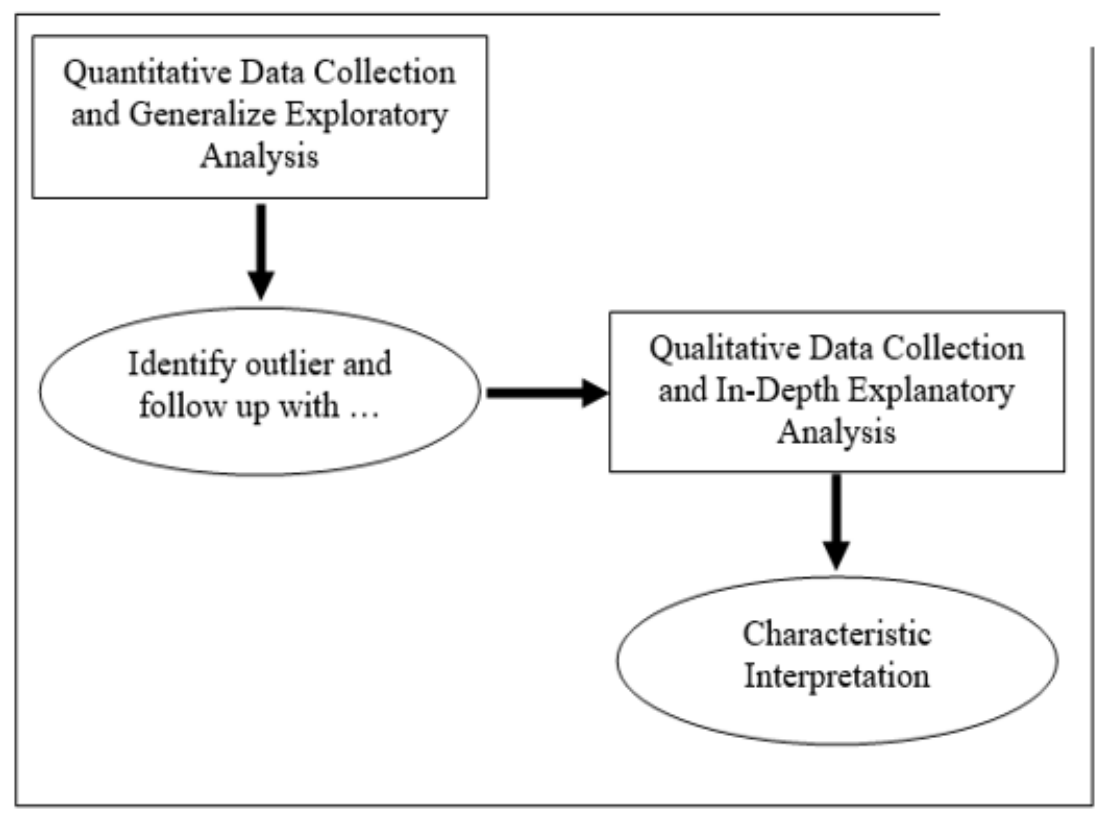

question also asking if the respondent has low satisfaction towards the store who they purchased the product from. The result of the following question was split into 3 categories and stated in the Figure 2 .

The second part of the questionnaire was asking about the respondent knowledge in virtual reality and especially

Figure 2. User Satisfaction

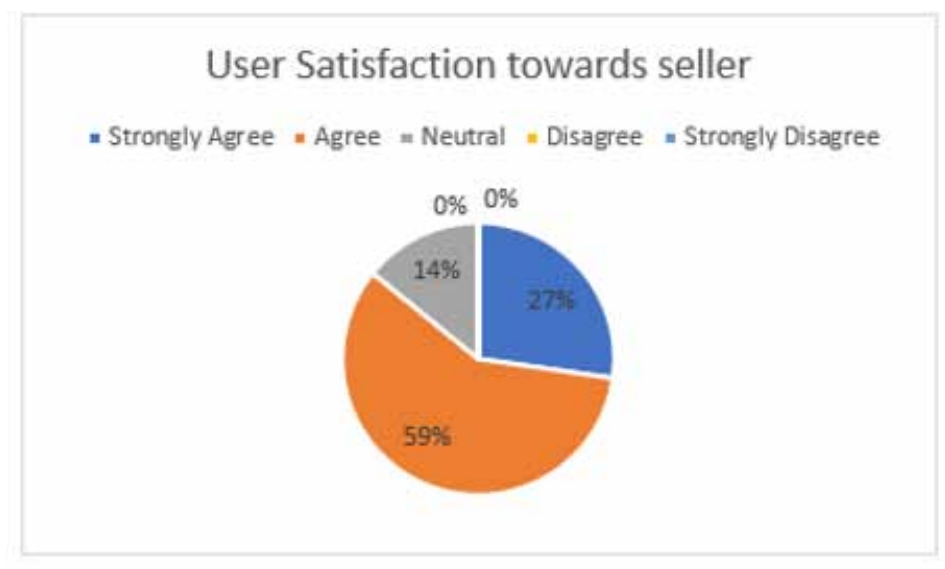


augmented reality which the study was discussing in shown in Figure 3. The respondent understanding of virtual reality technologies was great with $66 \%$ of the respondent knew about the technology, $27 \%$ of the respondent said they ever heard of virtual technologies, and only a small $7 \%$ of the respondent never heard of the technologies. The respondent knowledge in virtual reality technology was separated into 2 products which are augmented reality and virtual reality. The portion of respondent knowledge about virtual reality product shown in the diagram below. The diagram shows that $24 \%$ of the respondent mention that they know both augmented reality and virtual reality. Another $39 \%$ and $30 \%$ of the respondent only recognized either augmented reality or virtual reality. And the least part 7\% of the respondent do not know virtual reality technology. The conclusion for the second part of the questionnaire was the user already knew virtual reality technology especially augmented reality. Thus, the followed part of the question would be understandable by the respondent.

Figure 3. Understanding Virtual Reality Technology

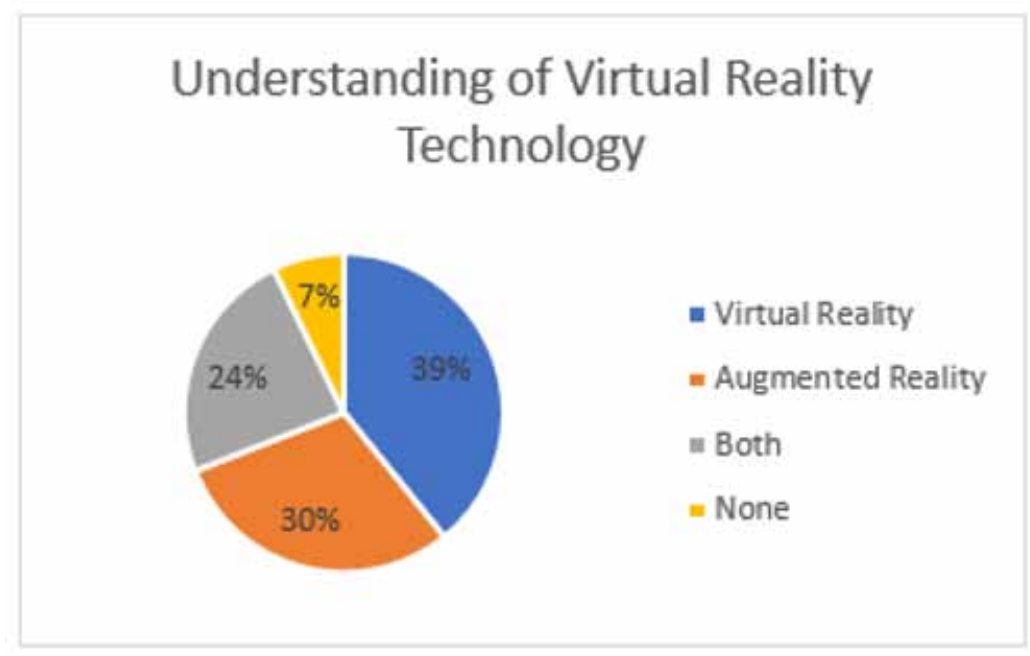

The last part of the questionnaire was discussed about implementation of augmented reality in the online shopping of furniture product as shown in Figure 4. The reason for the question was to be asking the respondent response in augmented reality implementation and opinion towards the user satisfaction. The question asked about the respondent preference in using the AR implemented online store. The response was predictable with the majority of the respondent answer yes that they wanted to see the augmented reality implementation in online shopping. Meanwhile, only a small percentage of the respondent answer that the implementation of the augmented reality was not important in the online shop experience.

The interview conducted through interviewing people on the online forum, interviewing people on google form, and interviewing people through voice call. The interview was quite surprising since more than half of the interviewees understand and agree with the augmented reality implementation. The other part of the interviewees rather giving suggestion for online furniture shopping or giving their experience in purchasing furniture.

The majority of interviewees who understand the augmented reality implementation giving insight that purchasing furniture online was quite tricky. The interviewees were aware that the product may be different from what they have seen in the online store. They even measure the product using a tape measure to make the furniture fit in their house. Unfortunately, not many people received the product 


\section{Augmented Reality Implementation}

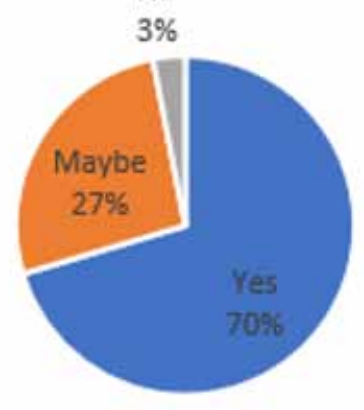

in the correct measurement. Some of the product may different some millimetres into centimetres. This, not a big problem for the purchasers since they already consider the issue before they purchase it. However, the augmented reality would provide better insight into the purchased product. And this would eliminate the needs of measuring the area before the purchase decision. The interviewees who had never faced any issue with purchasing furniture online would not consider measuring things at the beginning. They choose to see the furniture in augmented reality so that they can match the furniture with the surroundings.

The majority of the interviewees support the implementation of the augmented reality in furniture shopping since it could help to influence people consideration for buying the product. The interviewees also believed that augmented reality would increase customer satisfaction for buying the furniture online and would reduce the misunderstanding between the customer expectation and the product description.

From the data collection, the implementation of augmented reality in electronic commerce resulting in few requirements. Their requirements are the user able to use an e-commerce app, the user able to use augmented reality to project the image of the product.

The application would be separated into two part, first is the eCommerce part where the user can $\log$ in, view the product page and details, and purchase the product. The second is the place where the user uses the augmented reality to project an image of the product.

At the beginning of the application, the user will see the front page of the application. The front page of the application is the login page which the user needs to input the correct email and password. If the email and password are not registered in the application, it will not allow the user to enter the application. The snapshot below shows that the user will have the option to log in and register for the application. This page will verify the user credentials to enter the application itself. The application will not allow the user if the email is not registered in the database. The registered email will be reflected in the database.

After verifying the user email and password, the application will continue to the main menu part which will show the product in the application. The shown product is only for example for the study. The user will be able to choose the product and the application will open into product details. In this product details, the user will be able to see the details of the product. The snapshot above will show the application interface. The application interface will show the main menu and the product detail page. The figure shows the user interface of the e-commerce part. This electronic commerce part will be as simple as possible so that user can understand how each of the button work and every 
detail will state clearly. The user will be able to choose the product and the application will open into product details. In this product details, the user will be able to see the details of the product as shown in Figure 5.

\section{Figure 5. Application Dashboard}

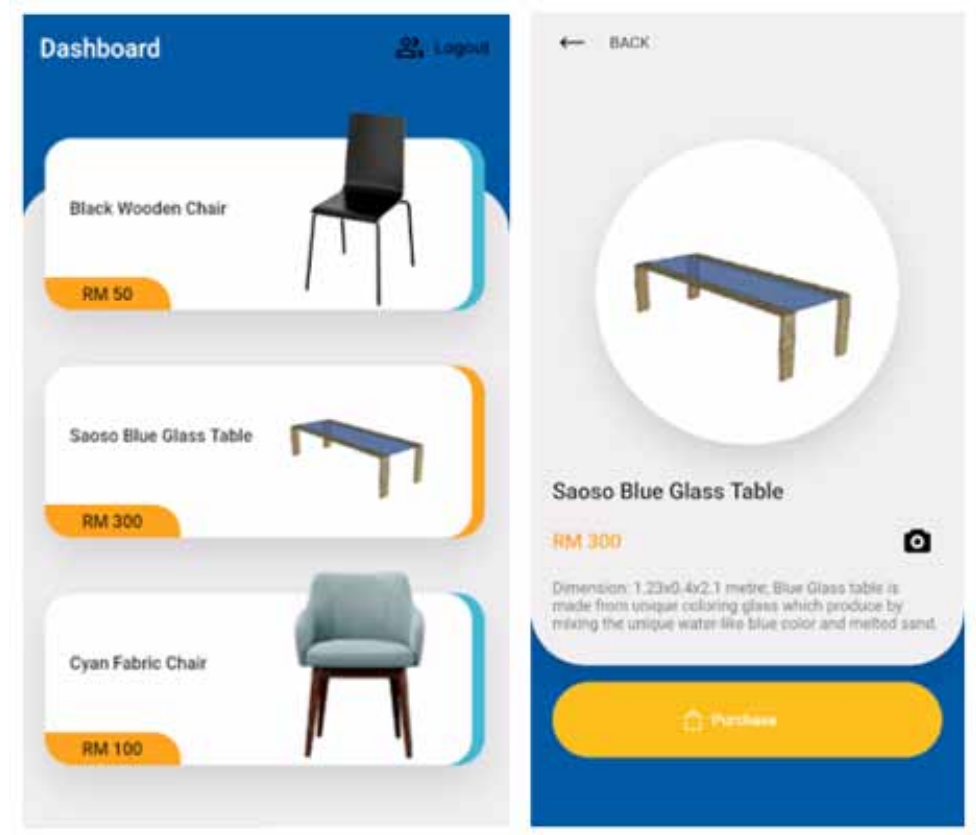

The augmented reality function can be seen on the details page. As there is a button to open the augmented reality system. The augmented reality system will be using unity and include the 3D model of the furniture. The beginning of augmented reality apps will be a sort of crosshair to place the object later. The object needs few seconds to scan the user surroundings. The user can place the crosshair wherever they want to. The crosshair will adapt to the user environment. The user can only place the object once the crosshair is placed and only need to touch the screen to place the object. Once the object placed on the user screen, they can see and move around to check the spawned object. The object as acts an immovable object so that the user can move around without the object following the user.

The figure 6 shows the snapshot of the augmented reality use for showing chair. The Augmented reality will allow the user to see the object from a 360-degree angle. As shown above, the blue coloured crosshair is where the object placed in the real-life surroundings and the object will spawn on top of the crosshair. The figure above also shows that the chair can be seen from many different angles and stay still regardless of the user position.

The user testing was resulting in fulfilling the user expectation. The user expects that they can see the dimension of the product in the details as well as in the augmented reality. The augmented reality will project the image as the user expectation. Even the user can see the product in 360-degree view and resulting in closer and more detail of the product. Furthermore, the user gives feedback in the augmented reality feedback which need some change to increase the user experience in using the augmented reality part. 

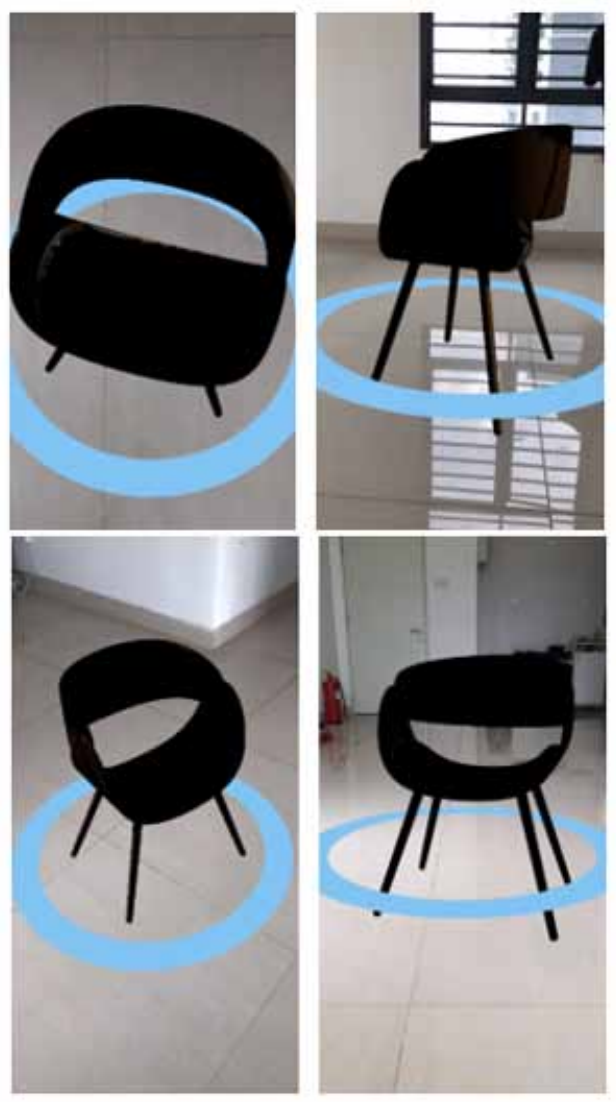

\section{CONCLUSION, LIMITATION, AND FUTURE WORK}

The study of the implementation of augmented reality in purchasing furniture concludes that the user can create the furniture object through augmented reality mobile application. The user also can understand the product and match it with their environment before they decide to buy the product. The augmented reality feature also become the main feature to improve the quality of furniture online shopping. The development of the application not only focus on augmented reality but also having an e-commerce feature so that the user makes use of it.

The recommendation for future study would be an additional feature in augmented reality since the study only covers furniture implementation in augmented reality. Future study may want to use augmented reality as a feature to be implemented in other smaller product such as clothing and small gadget. Moreover, the future study can use the augmented reality not only in electronic commerce but also in an industrial area or working area that needs projection for the product. 


\section{REFERENCES}

AdrianChin, Y.K., JosephNg, P. S., Shibghatullah, A. S., \& Loh, Y. F. (2020). JomDataMining: Learning behaviour affecting their academic performance, really? IEEE 6th International Conference on Engineering Technologies and Applied Science.

AdrianChin, Y.K., JosephNg, P.S., Eaw, H.C., Loh, Y.F. \& Shibghatullah, A.S. (2020). JomDataMining: Academic Performance and Learning Behavior Dubious Relationship. International Journal of Business Information Systems.

Beck, J., Rainoldi, M., \& Egger, R. (2019). Virtual reality in tourism: A state-of-the-art review. Tourism Review, 74(3), 586-612. doi:10.1108/TR-03-2017-0049

Chang, I.C., Liu, C.C., \& Chen, K. (2014). The effects of hedonic/utilitarian expectations and social influence on continuance intention to play online games. Internet Research, 24(1), 21-45.

Chiu, W., \& Cho, H. (2019). E-commerce brand: The effect of perceived brand leadership on consumers' satisfaction and repurchase intention on e-commerce websites. Asia Pacific Journal of Marketing and Logistics.

Cowan, K., \& Ketron, S. (2019). Prioritizing marketing research in virtual reality: Development of an immersion/ fantasy typology. European Journal of Marketing, 53(8), 1585-1611.

Domina, T., Lee, S.-E., \& MacGillivray, M. (2012). Understanding factors affecting consumer intention to shop in a virtual world. Journal of Retailing and Consumer Services, 19, 613-620.

Engelmann, T., Wallstein, S., \& Hitzler, D. (2019). An Experimental Study to Investigate the Potential of Online Shopping in Immersive Virtual Realities Compared to Conventional Online Shops. The International Journal of Virtual Reality: a Multimedia Publication for Professionals, 19(3), 31-45. doi:10.20870/IJVR.2019.19.3.2939

Gupta, P., \& Dubey, A. (2017). Franchising and e-commerce a realistic business option: An analytical study of Intex Smart World. International Journal of Advanced Research in Computer Science, 8(3), 555-563.

Haenlein, M., \& Kaplan, A. M. (2009). Flagship brand stores within virtual worlds: The impact of virtual store exposure on real-life attitude toward the brand and purchase intent. Recherche et Applications en Marketing, 24(3), 57-79. doi:10.1177/076737010902400304

Hussy, W., Schreier, M., \& Echterhoff, G. (2015). Forschungsmethoden in Psychologie und Sozialwissenschaften für Bachelor. Springer.

Javornik, A. (2016b). Augmented reality: Research agenda for studying the impact of its media characteristics on consumer behaviour. Journal of Retailing and Consumer Services, 30, 252-261. doi:10.1016/j. jretconser.2016.02.004

JosephNg, Kang, Choo, Wong, Phan, \& Lim. (2015). Beyond cloud infrastructure services in medium-size manufacturing. International symposium on mathematical sciences \& computing research, 150-155.

JosephNg, Kang, Ahmad, Wong, Phan, Saw, \& Lim. (2016). EaaS: Available yet Hidden Infrastructure inside MSE. 5th International Conference on Network, Communication and Computing, ACM International Conference Proceeding Series, 17-20.

JosephNg, Kang, Mahmood, Choo, Wong, Phan, \& Lim. (2016). Exostructure Services for Infrastructure Resources Optimization. Journal of Telecommunication, Electronic \& Computer Engineering, 8(4), 65-69.

JosephNg. (2018). EaaS Optimization: Available yet hidden information technology infrastructure inside mediumsize enterprises. Journal of Technological Forecasting and Social Change, 132(July), 165-173.

JosephNg. (2019). EaaS Infrastructure Disruptor for MSE. International Journal of Business Information Systems, 30(3), 373-385.

JosephNg, Loh, \& Eaw. (2020). Grid Computing for MSE during Volatile Economy. International Conference on Control, Automation and Systems, 709-714.

JosephNg \& Eaw. (2021). Making Financial Sense from EaaS for MSE during Economic Uncertainty. Springer Advances in Intelligent Systems and Computing. 
JosephNg, P. S., Choo, P. Y., Wong, S. W., Phan, K. Y., \& Lim, E. H. (2012). Hibernating ICT Infrastructure During Rainy Days. Journal of Emerging Trends in Computing \& Information Sciences, 3(1), 112-116.

JosephNg, P. S., Mahmood, A. K., Choo, P. Y., Wong, S. W., Phan, K. Y., \& Lim, E. H. (2013). Battles in volatile information and communication technology landscape: The Malaysia small and medium enterprise case. International Journal of Business Information Systems, 13(2), 217-234.

JosephNg, P. S., Mahmood, A. K., Choo, P. Y., Wong, S. W., Phan, K. Y., \& Lim, E. H. (2014). IaaS Cloud Optimization during Economic Turbulence for Malaysia Small and Medium Enterprise. International Journal of Business Information Systems, 16(2), 196-208.

JosephNg, P. S., Mahmood, A. K., Choo, P. Y., Wong, S. W., Phan, K. Y., \& Lim, E. H. (2015). Barebone cloud IaaS: Revitalization disruptive technology. International Journal of Business Information Systems, 18(1), 107-126.

JosephNg \& Eaw. (2021). Still Technology Acceptance Model? Reborn: Exostructure as a Service Model. International Journal of Business Information Systems.

JosephNg \& Kang. (2016). Beyond barebone cloud infrastructure services: Stumbling competitiveness during economic turbulence. Journal of Science and Technology, 24(1), 101-121.

Kang, C. M., JosephNg, P.S., \& Issa, K. (2015), A study on integrating penetration testing into the information security framework for Malaysian higher education institutions. International symposium on mathematical science \& computing research, 156-161. doi:10.1109/ISMSC.2015.7594045

Liow, K. M. (2021). JomMachineLearning: Bringing Artwork Nearer with DesignLab. International Journal of Business Strategy and Automation, 2(2), 54-71.

Malaysian Communications and Multimedia Commission. (2018). e-Commerce Consumer Survey 2018. Author.

JosephNg, Choo, Wong, Phan, \& Lim. (2012). Malaysia SME ICT During Economic Turbulence. International Conference on Information \& Computer Network, 67-71.

JosephNg, Yin, Wan, \& Nazmudeen. (2011). Energizing ICT Infrastructure for Malaysia SME during Economic Turbulence. Student Conference on Research and Development, 328-322.

Reynolds, J. (2015). eCommerce: A critical review. International Journal of Retail \& Distribution Management, 28(10), 417-444. doi:10.1108/09590550010349253

Roxo, M. T., \& Brito, P. Q. (2018). Augmented Reality Trends to the Field of Business and Economics: A Review of 20 years of Research. Asian Journal of Business Research, 8(2), 94-117.

Seidl, D. (2018). Handel im Wandel durch virtuelle und erweiterte Realitäten [Changing through virtual and expanded realities]. In M. Knoppe \& M. Wilde (Eds.), Digitalisierung im Handel (pp. 223-233). Academic Press.

Trabelsi-Zoghlami, A., \& Touzani, M. (2019). How real are virtual experiences? For a better understanding of virtual experiences and their impact on consumers' real life. European Journal of Marketing, 53(8), 1612-1636. doi:10.1108/EJM-10-2017-0776

Vernette, E. (2018). Comprendre et interpréter une expérience de consommation avec l'Album on-Line (AOL): une application au surf des MERS. 7th International Congress Marketing Trends, Venice, Italy.

Vica, V. (2020). JomImage SnapFudo: Control your Food in a Snap. International Conference on Engineering Technologies and Applied Sciences, 1-5.

Vivilyana, V. (2020). JomImage: Weight Control with Mobile SnapFudo. Advances in Intelligent Systems and Computer, 1252(3), 168-180.

Wakil, K., Alyari, F., Ghasvari, M., Lesani, Z., \& Rajabion, L. (2019). A new model for assessing the role of customer behaviour history, product classification, and prices on the success of the recommender systems in e-commerce. Kybernetes.

Watson, A., Alexander, B., \& Salavati, L. (2018). The impact of experiential augmented reality applications on fashion purchase intention. International Journal of Retail \& Distribution Management. 
Yaoyuneyong, G., Foster, J., Johnson, E., \& Johnson, D. (2016). Augmented reality marketing: Consumer preferences and attitudes toward hypermedia print ads. Journal of Interactive Advertising, 16(1), 16-30. doi:1 $0.1080 / 15252019.2015 .1125316$

Yoo, S. C., \& Eastin, M. S. (2016). Contextual advertising in games: Impacts of game context on a player's memory and evaluation of brands in video games. Journal of Marketing Communications, 614-631. 